\title{
Editorial: Special Issue on "Recent Advances in Mobile and Wireless Networks"
}

\author{
Der-Jiunn Deng ${ }^{1}$ - Chih-Cheng Tseng ${ }^{2}$ Tony Q. S. Quek ${ }^{3}$ • \\ Nirwan Ansari ${ }^{4}$
}

Published online: 18 August 2015

(C) Springer Science+Business Media New York 2015

Mobile and wireless networks are enabling a myriad of services and applications. For examples, wireless hotspots are so widely deployed that access to the Internet is almost ubiquitous; people can work and play via Internet access no matter whether they are sitting on a terrace, travelling on a train, or flying in a plane. The explosive growth of applications for the current and emerging user services poses a number of challenges to this critical infrastructure. It also presents opportunities for research and innovation to both the academia and industry, especially for the development of efficiency, scalability and reliability in mobile and wireless networks. These challenges motivate us to design and develop new protocols, architectures and services for future mobile and wireless networks.

The goal of this special issue is to publish both state-of-the-art and prospective papers that present advances towards the performance enhancement of mobile and wireless networks. The call for papers for this special issue attracted 107 submissions from Asia, Africa, Europe, and the US covering a wide range of topics in the related field. Each paper was carefully evaluated by at least three reviewers. This careful evaluation process has

Der-Jiunn Deng

djdeng@cc.ncue.edu.tw

Chih-Cheng Tseng

tsengcc@niu.edu.tw

Tony Q. S. Quek

tonyquek@sutd.edu.sg

Nirwan Ansari

nirwan.ansari@njit.edu

1 National Changhua University of Education, Bao-Shan Campus, No. 2, Shi-Da Rd, Changhua City 500, Taiwan, ROC

2 National Ilan University, No. 1, Sec. 1, Shen Lung Road, Yilan 26041, Taiwan, ROC

3 Singapore University of Technology and Design, 8 Somapah Road, Singapore 487372, Singapore

4 New Jersey Institute of Technology, University Heights, Newark, NJ 07102, USA 
allowed us to select 29 high quality research papers. We strongly believe that the selected papers will make a significant contribution to this field. We present a brief overview of each manuscript in the following.

The 29 accepted papers are divided into four categories. Eleven papers are related to MAC and cognitive networks. In the paper entitled "On Optimal Threshold Selection in Cooperative Spectrum Sensing for Cognitive Radio Networks- an Energy Detection Approach using Fuzzy Entropy Maximization" authored by S. Chatterjee, et al., an optimization problem is formulated to determine multiple threshold values for cooperative spectrum sensing in cognitive radio networks. Fuzzy entropy maximization on sensed energy is suggested as solution. "A Quality-of-Service Routing Protocol with Supplementary Cooperation for Wireless Ad Hoc Networks" by S. L. Su et al. proposes a spatialreuse time-division multiple access (STDMA)-based QoS routing protocol to provide the service guarantee of target data rate and maximum end-to-end outage probabilities for data flows in the multihop ad hoc networks. "Energy-Efficient Design of Sensing and Transmission in Cognitive Radio Networks" by X. Feng et al. designs an energy efficient sensing and transmission strategy for a secondary user (SU) in a cognitive radio network. "Bursty-Contention Distribution for Energy Efficiency in Large Scale IEEE 802.15.4 Wireless Sensor Networks" by H. R. Lee et al. proposes a new and simply implementable contention mitigation scheme to enhance the energy efficiency of sensor nodes by reducing the contention degree among neighboring coordinators. "Efficient Internet Access Framework for Mobile Ad Hoc Networks" by R. Attia et al. proposes an efficient Adaptive Distributed Multipath Internet GateWays (ADMIGW) framework that considers the main challenges encountered in the MANET-Internet integration. "Fast shared Feedback Methods for Uplink CoMP with H-ARQ" by Y. H. Jung et al. proposes a direct feedback method from multiple coordinated base stations to a mobile station that uses a shared feedback channel for an uplink coordinated multipoint operation with hybrid automaticrepeat request (H-ARQ) transmission. "Cognitive Network Management Framework and Approach for Video Streaming Optimization in Heterogeneous Networks" by T. Ojanperä et al. proposes a network management framework that relies on cognitive decision techniques in the joint optimization of network and video service performance. "Adaptive Quorum-Based Power-Saving Protocol for Asynchronous Wireless Ad Hoc Networks" by Y. S. Chen et al. proposes an adaptive power-saving protocol for asynchronous wireless ad hoc networks in which the sleep-awake patterns of the wireless devices are determined by using a grid quorum-based approach. "Green and Cooperative DASH in Wireless D2D Networks" by L. Zhang et al. proposes a D2D DASH framework to promote cooperation among devices, while enhancing video quality of service. "An Energy-Efficient User Location-Aware Switch-Off Method for LTE-A Cellular Networks" by X. Su et al. proposes a distributed switch-off method, based on the User Equipment locations and minimax algorithm, for the Orthogonal Frequency Division Multiplexing Access (OFDMA)based cellular networks. "A Survivability Clustering Algorithm for Ad Hoc Network Based on a Small-World Model" by W. Zhang et al. proposes a new algorithm, EMDWCA, to effectively extend the lifetime of an Ad hoc network.

Six papers are about PHY and wireless communications. "Performance Analysis of Hierarchically Combined Practical Beamforming Methods" by B. B. Haile et al. introduces a hierarchical beamforming structure whereby a combination of beamforming methods can be applied using either co-located or distributed antenna systems. In the paper entitled "Buffer-Aware and Delay-Sensitive Resource Allocation in the Uplink of 3GPP LTE Networks" authored by C. Wang et al., resource allocation problems in uplink transmissions of long term evolution (LTE) networks involving single-carrier frequency division 
multiple access (SC-FDMA) were addressed and a two-stage process for obtaining solutions was proposed. In the paper entitled "Automatic Modulation Classification for Low SNR Digital Signal in Frequency-Selective Fading Environment" authored by W. Wallayt et al., the Maximum Likelihood classifier for the Automatic Modulation Classification of PSK and the QAM modulated digital signals in frequency selective fading channel along with the addition of Non-Gaussian noise has been proposed. "Iterative Channel Estimation and Detection for High-Mobility MIMO-OFDM Systems: Mitigating Error Propagation by Exploiting Error Information" by R. Yang et al. proposes a new iterative channel estimation and detection scheme that reduces both detection errors and channel estimation errors. "Full-Duplex Massive MIMO Relaying: An Energy Efficiency Perspective" by Y. $\mathrm{Xu}$ et al. shows that the power control problem can be approximated as a geometric program problem and solved efficiently by the convex optimization tools. "Offloading Multiple Mobile Data Contents through Opportunistic Device-to-device Communications" by R. G. Cheng et al. presents a popularity-based relaying user selection algorithm to determine the number of relaying users for distributing multiple contents with different popularity.

Six papers address security and authentication issues. In the paper entitled "ES-AKA: An Efficient and Secure Authentication and Key Agreement Protocol for UMTS Networks" authored by N. Saxena et al., an authentication and key agreement (AKA) protocol of Universal Mobile Telecommunication System (UMTS) was proposed to solve the vulnerabilities found in Global System for Mobile Communications (GSM) systems. "Provably secure and anonymous password authentication protocol for roaming service in global mobility networks using extended chaotic maps" by S. H. Islam et al. puts forwarded a new provably secure and privacy-preserving password authentication protocol by using extended chaotic maps for roaming service in wireless networks. "An Efficient Pairwise and Group Key Management Protocol for Wireless Sensor Network" by M. Rahman et al. proposes an efficient key management protocol for WSNs that provides support for both pairwise and group-wise key management. "A Secure Authentication Scheme with User Anonymity for Roaming Service in Global Mobility Networks" by M. Karuppiah et al. proposes a secure authentication scheme with user anonymity for roaming service in global mobility networks. "Analyzing Three-Fold Schemes for Enhancing Communication Channel Efficiencies Using IP Multimedia Server-Client Systems for LTE Networks" by B. D. Deebak et al. proposes three novel schemes to resolve the issues of IP Multimedia Subsystem (IMS) and integrate the proposed and existing authentication schemes in the IMS server and client environments to verify the metrics. "An AttributeRole Based Access Control Mechanism for Multi-Tenancy Cloud Environment” by N.W. Lo et al. improves the role-based access control authorization mechanism and, by combining it with attribute check mechanism, can determine which tenant that user can access. The enhanced authorization mechanism can improve the safety of cloud computing services and protected the data privacy.

The last six papers are related to services and applications. "Utilizing the Domain Name System for Scalable and Secure Named Data Networking” by Z. Yan et al. proposes a new scheme that utilizes current DNS protocols for content naming and addressing in a NDN environment. "ICA: An Incremental Clustering Algorithm Based on the OPTICS" by J. $\mathrm{S}$. Fu et al. proposes an incremental clustering algorithm (ICA) based on OPTICS (Ordering points to identify the clustering structure). "An Effective NAT Traversal Mechanism for SIP/IMS Services in SDN-enabled All-IP Mobile Networks" by W. E. Chen et al. integrates Software-defined networking (SDN) into the All-IP mobile networks and proposes a solution to improve the ALG solution in the SDN-enabled All-IP 
mobile networks. "A specific heuristic dedicated to a coverage/tracking bi-objective problem for wireless sensor deployment" by M. L. Berre et al. proposes a specific heuristic (H3P) based on the mathematical decomposition of the problem and the clustering of localization variables. "Resource-Optimal Heterogeneous Machine-to-Machine Communications in Software Defined Networking Cyber-Physical Systems" by S. Y. Lien develops mathematical foundations of a resource-optimal design for Cyber-physical systems, and achieves the minimum resource usage to support real-time data exchanges. Finally, the special issue concludes with an article entitled "State-of-the-Art Business Performance Evaluation of the Advanced Wireless Heterogeneous Networks to be deployed for the "TERA age" authored by V. Nikolikj et al. In this paper, the authors presented a conceptual model to assess the business performance of the future advanced wireless and mobile heterogeneous network.

As the guest editors of this special issue, we would like to thank all authors who have submitted papers to the special issue and in particular those whose papers have been accepted for this special issue. Assistance from the editorial staff of the Wireless Personal Communications is also much appreciated. Besides, the guest editors wish to acknowledge all those who have generously given their time to review the papers submitted for consideration for inclusion in this special issue. Finally, our special thanks go to Professor Ramjee Prasad (editor-in-chief) for his valuable support throughout the preparation of this special issue.
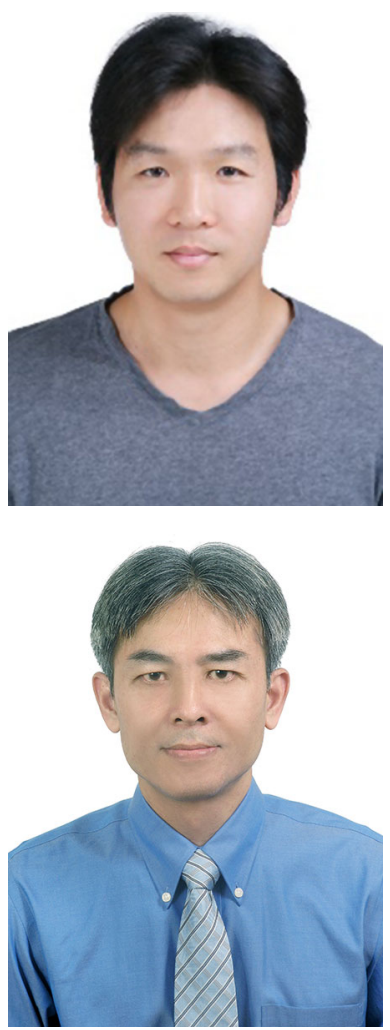

Der-Jiunn Deng received the Ph.D. degree in electrical engineering from the National Taiwan University in 2005. He joined the National Changhua University of Education as an assistant professor in the Department of Computer Science and Information Engineering in August 2005 and then became a full professor in February 2012. His research interests include multimedia communication, quality-of-service, and wireless networks. In 2010, 2011, and 2012, he received the Research Excellency Award of National Changhua University of Education. In 2012, he also received the Outstanding Faculty Research Award of National Changhua University of Education. Dr. Deng served or is serving as an editor and guest editor for several technical journals. He also served or is serving on several symposium chairs and technical program committees for IEEE and other international conferences. Dr. Deng is a member of the IEEE.

Chih-Cheng Tseng received his B.S. and M.S. from the National Taiwan University of Science and Technology, Taipei, Taiwan, Republic of China, in 1994 and 1997 respectively, all in electronic engineering. He received his Ph.D. from the Graduate Institute of Communications Engineering, National Taiwan University, Taipei, Taiwan, Republic of China, in 2007. He is currently an associate professor of the Department of Electrical Engineering, National Ilan University, Yi-Lan, Taiwan, Republic of China. He was a visiting researcher at the Center for TeleInFrastruktur (CTIF), Aalborg University, Denmark on 2007 summer. His research interests include the design and performance evaluation of protocols for the next generation mobile communications and wireless ad hoc/sensor networks. $\mathrm{He}$ is a member of IEEE. 


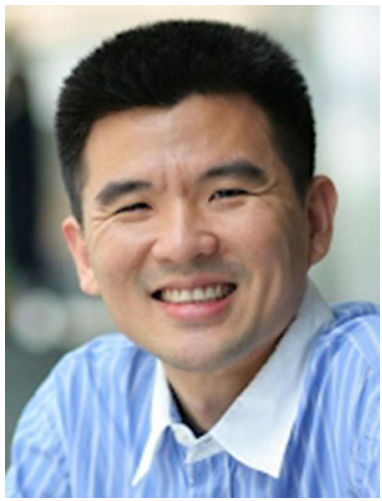

Tony Q. S. Quek is an Assistant Professor with the Singapore University of Technology and Design (SUTD), leading the Wireless Networks and Decision Systems (WNDS) Group. He is also a Scientist with the Institute for Infocomm Research, A*STAR. He received the B.E. and M.E. degrees in Electrical and Electronics Engineering from Tokyo Institute of Technology, respectively. At MIT, he earned the Ph.D. in Electrical Engineering and Computer Science. He is a senior member of the IEEE. His main research interests are the application of mathematical, optimization, and statistical theories to communication, networking, signal processing, and resource allocation problems. Current research topics include heterogeneous networks, green communications, smart grid, security, big data processing, IoT, and cognitive radio.

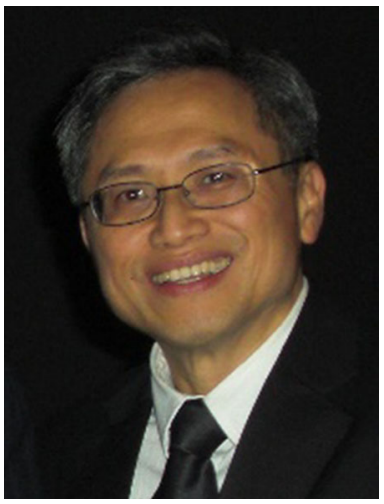

Nirwan Ansari received the B.S.E.E. degree (summa cum laude) from the New Jersey Institute of Technology (NJIT), Newark, NJ, USA, the M.S.E.E. degree from the University of Michigan, Ann Arbor, MI, USA, and the Ph.D. degree from Purdue University, West Lafayette, IN, USA. He is Distinguished Professor of Electrical and Computer Engineering at NJIT, which he joined in 1988. He has also assumed various administrative positions at NJIT. He has been a Visiting (Chair) Professor at several universities. He authored Media Access Control and Resource Allocation (Springer, 2013) with J. Zhang and Computational Intelligence for Optimization (Springer, 1997) with E.S.H. Hou, and edited Neural Networks in Telecommunications (Springer, 1994) with B. Yuhas. He has also contributed over 450 technical papers, over one-third of published in widely cited refereed journals/magazines. He has guest edited a number of Special Issues, covering various emerging topics in communications and networking. His current research focuses on green communications and networking, cloud computing, and various aspects of broadband networks. He has served on the Editorial Board and Advisory Board of over ten journals, including as a Senior Technical Editor of IEEE Communications Magazine (2006-2009). He was elected to serve on the IEEE Communications Society (ComSoc) Board of Governors as a Member-at-Large (2013-2015). He has chaired ComSoc Technical Committees, and has actively organized numerous IEEE international conferences/symposia/workshops, assuming various leadership roles. Some of his recognitions include several Excellence in Teaching Awards, two Best Paper Awards, Purdue University Outstanding Electrical \& Computer Engineer Award (2015), NCE Excellence in Research Award (2014), ComSoc AHSN TC Outstanding Service Recognition Award (2013), NJ Inventors Hall of Fame Inventor of the Year Award (2012), Thomas Alva Edison Patent Award (2010), and designation as an IEEE Communications Society Distinguished Lecturer (2006-2009, two terms). He has also been granted over 25 U.S. patents. 